TRANSACTIONS OF THE

AMERICAN MATHEMATICAL SOCIETY

Volume 352, Number 9, Pages 3957-3969

S 0002-9947(00)02481-8

Article electronically published on May 22, 2000

\title{
SOME PROPERTIES OF MINIMAL SURFACES IN SINGULAR SPACES
}

\author{
CHIKAKO MESE
}

\begin{abstract}
This paper involves the generalization of minimal surface theory to spaces with singularities. Let $X$ be an NPC space, i.e. a metric space of non-positive curvature. We define a (parametric) minimal surface in $X$ as a conformal energy minimizing map. Using this definition, many properties of classical minimal surfaces can also be observed for minimal surfaces in this general setting. In particular, we will prove the boundary monotonicity property and the isoperimetric inequality for minimal surfaces in $X$.
\end{abstract}

\section{INTRODUCTION}

Minimal surface theory has been an extremely active and fruitful area of research for more than two centuries. During the first part of this century, the main emphasis was placed on the existence of the solution to the Plateau Problem in $\mathbf{R}^{n}$. Since then, there has been various work investigating the geometric properties of minimal surfaces. Moreover, numerous generalizations of the theory have taken place; for example, to higher dimensions, to Riemannian spaces and to wider classes of surfaces. Our present paper involves the generalization of minimal surface theory to spaces with singularities.

Our study is motivated by the successful study of harmonic maps into singular spaces which was initiated by Gromov and Schoen in [GS]. Their analysis of harmonic maps into Riemannian simplicial complexes combined with Corlette's vanishing theorem $[\mathrm{C}$ is the basis of their proof of $p$-adic super-rigidity for lattices in $S P(n, 1)$ and $F_{4}$. The study of harmonic maps was further generalized in [KS1, KS2], and independently by Jost [J1]. Harmonic map theory in singular spaces have proven to be useful in answering many questions including the rigidity question mentioned above as well as others (for example [J2]). Other examples include Wolf's [W1], W2 investigations of Teichmüller spaces and the actions of fundamental groups of closed surfaces and Hardt and Lin's [HL] study of neumatic liquid crystals.

The spaces we consider are NPC spaces, i.e., metric spaces with non-positive curvature where the curvature condition is defined in terms of Toponogov's triangle comparison: geodesic triangles are required to be thinner than comparison triangles in $\mathbf{R}^{2}$. (See Section 2 for a precise definition.) This notion of curvature was developed by A.D. Alexandrov and the Russian school of mathematicians in the 1940's and 1950's in order to give a synthetic, coordinate-free description of Riemannian spaces (see for example, $[\mathrm{ABN}]$ ). They include smooth Riemannian

Received by the editors March 24, 1998 and, in revised form, November 1, 1998.

1991 Mathematics Subject Classification. Primary 58E12.

(C)2000 American Mathematical Society 
manifolds and manifolds with cone singularities as well as spaces that are not topological manifolds such as a Riemannian simplicial complex. In fact, no restriction is made on the types of singularities allowed in its definition.

Nikolaev [N] was the first to consider minimal surface theory in this setting and gave the solution to the Plateau Problem. Following the variational methods developed by Korevaar and Schoen, we consider a different definition of the area than that by Nikolaev. This allows us to follow the well-known classical approach (see [Mo]) to obtain the solution to the Plateau Problem. The solution obtained in this way is a harmonic (energy minimizing) and weakly conformal map. Since a minimal surface in a smooth space has a parameterization which is harmonic and conformal, we define minimal surfaces in this setting to be maps which are harmonic and weakly conformal. In [Me1, we show that the curvature of minimal surfaces in a space with curvature bounded from above by $\kappa$ is also bounded from above by the same constant, generalizing a well-known fact regarding minimal surfaces in a smooth Riemannian manifold.

In this paper, we will generalize several additional properties of minimal surfaces which are well-known in the classical setting. We will show that if $u: M \rightarrow X$ is a minimal surface from a smooth compact Riemannian surface $M$ with boundary $\partial M$ to an NPC space $X$, then

Theorem A. The map u cannot tend to a single point along any boundary arc of $M$.

Theorem B. There is a universal constant $C$ so that $L^{2} \geq C A$ where $L$ is the length of the boundary and $A$ is the area of minimal surface.

In the classical case, the coordinate functions of a conformal parameterization $u: \Omega \subset \mathbf{R}^{2} \rightarrow \mathbf{R}^{n}$ of a minimal surface are harmonic functions. This combined with the conformality of the map can be used to show that the boundary map is monotonic. Many proofs of the isoperimetric inequality with $C=4 \pi$ exist for minimal surfaces in $\mathbf{R}^{n}$ with various assumptions on the configuration of the boundary. Michael and Simon [MS] gave a proof which handles all minimal surfaces (actually their proof works in higher dimensions and minimal submanifolds) but with $C<4 \pi$.

The key element in the proof of Theorem A is the convexity of the distance function in the NPC setting. The isoperimetric inequality is proved by using the argument of Michael and Simon. We note that the conjectured best constant for $C$ is $4 \pi$, but this is still unsolved even for minimal surfaces in $\mathbf{R}^{3}$ without additional assumptions.

\section{ACKNOWLEDGMENTS}

The author wishes to thank the referee for many valuable suggestions. They were particularly helpful in shortening many arguments and improving the exposition of the paper. She also thanks Professor Richard Schoen and Professor Paul Yang for their interest in this work.

\section{Preliminaries}

2.1. Metric Spaces of Curvature Bounded from Above. We review the notion of curvature bounds in a metric space $(X, d)$. (We will use the shorthand notation of writing $d_{P Q}$ instead of $d(P, Q)$.) First, we assume our metric spaces to 
be length spaces, i.e. for each $P, Q \in X$, there exists a curve, which we denote $\overline{P Q}$, such that the length of $\overline{P Q}$ is exactly $d_{P Q}$. We call $\overline{P Q}$ a geodesic between $P$ and $Q$. We then say that $X$ is an NPC space (also referred to as CAT(0) spaces in literature) or that $X$ has non-positive curvature in the sense of Alexandrov if geodesic triangles in $X$ are thinner than their comparison triangles in $\mathbf{R}^{2}$. In other words, for every $P, Q, R \in X$ and corresponding points $\bar{P}, \bar{Q}, \bar{R} \in \mathbf{R}^{2}$ with

$$
d_{P Q}=|\bar{P}-\bar{Q}|, d_{Q R}=|\bar{Q}-\bar{R}|, d_{R P}=|\bar{R}-\bar{P}|,
$$

we have

$$
d_{P Q_{t}} \leq\left|\bar{P}-\bar{Q}_{t}\right|
$$

where $Q_{t}$ is the point of $\overline{P Q}$ and $\bar{Q}_{t}$ is the point of the line segment between $\bar{R}$ and $\bar{Q}$ so that $d_{P Q_{t}}=t d_{P Q}$ and $\left|P-Q_{t}\right|=t|P-Q|$. By calculating $\left|\bar{P}-\bar{Q}_{t}\right|$, we have a more explicit formula:

$$
d_{P Q_{t}}^{2} \leq(1-t) d_{P Q}^{2}+t d_{P R}-t(1-t) d_{Q R}
$$

The more general notion of curvature bounded from above by some constant $\kappa$ is defined by replacing $\mathbf{R}^{2}$ with surface $S_{\kappa}$ of constant curvature $\kappa$ and requiring that $d_{P Q}+d_{Q R}+d_{R P}<\frac{\pi}{\sqrt{\kappa}}$ if $\kappa>0$. (See $[\mathrm{ABN}]$ for equivalent definitions.) Furthermore, we have

Definition 2.1. Let $\gamma, \sigma$ be geodesics in $X$ emanating from point $P$. Then

$$
\alpha(\gamma, \sigma)=\lim _{t, s \rightarrow 0} \arccos \frac{d^{2}(\gamma(t), P)+d^{2}(\sigma(s), P)-d^{2}(\gamma(t), \sigma(s))}{2 d(\gamma(t), P) d(\sigma(s), P)}
$$

is called the angle between $\gamma$ and $\sigma$.

The limit above exists because $X$ is an NPC space. Since geodesics are unique in an NPC space, the above definition implies that for $P, Q, R \in X$ we can define

$$
\angle(Q P R)=\alpha(\overline{P Q}, \overline{P R})
$$

The following important result is given in $[\mathrm{R} 2]$.

Theorem 2.2 (Reshetnyak). Let $(X, d)$ be a metric space of curvature bounded from above by $\kappa$ and $\Gamma$ be a closed rectifiable curve in $X$ with length less than $\frac{\pi}{\sqrt{\kappa}}$ if $\kappa>0$. Then there exists a convex domain $V$ in $S_{\kappa}$ and a map $\varphi: V \rightarrow X$ such that $\varphi(\partial V)=\Gamma$, the lengths of the corresponding arcs coincide, and $d_{S_{\kappa}}(x, y) \geq$ $d(\varphi(x), \varphi(y))$, for $x, y \in V$.

In particular, this implies that for an ordered sequence $\{P, Q, R, S\} \subset X$, there exists an ordered sequence $\{\bar{P}, \bar{Q}, \bar{R}, \bar{S}\} \subset \mathbf{R}^{2}$ which forms the consecutive vertices of a convex quadrilateral and which satisfies

$$
\begin{array}{rlrl}
d_{P Q} & =|\bar{P}-\bar{Q}|, & d_{Q R}=|\bar{Q}-\bar{R}|, \\
d_{R S}=|\bar{R}-\bar{S}|, & d_{S P}=|\bar{S}-\bar{P}|, \\
d_{P R} \leq|\bar{P}-\bar{R}|, & d_{Q S} \leq|\bar{Q}-\bar{S}| .
\end{array}
$$

We call $\{\bar{P}, \bar{Q}, \bar{R}, \bar{S}\}$ the subembedding of $\{P, Q, R, S\}$. In particular, we see that

$$
\begin{array}{rlrl}
\angle(P Q R) & \leq \angle(\bar{P} \bar{Q} \bar{R}), & & \angle(Q R S) \leq \angle(\bar{Q} \bar{R} \bar{S}), \\
\angle(R S P) \leq \angle(\bar{R} \bar{S} \bar{P}), & & \angle(S P Q) \leq \angle(\bar{S} \bar{P} \bar{Q}) .
\end{array}
$$


2.2. Variational Theory in Complete Metric Spaces. In KS1, Korevaar and Schoen develop the Sobolev space theory for maps into complete metric spaces. A map $u: \Omega \rightarrow X$ from a Riemannian domain to a complete metric space is in $L^{2}(\Omega, X)$ if for some point $Q \in X, d(u(x), Q)$ is in $L^{2}(\Omega)$. The energy density $|\nabla u|^{2}(x)$ and norm of directional derivative $\left|u_{*}(V)\right|(x)$ for $V \in \Gamma(T \bar{\Omega})$ are generalized in this setting. The energy of the map $u$ is defined as

$$
E(u)=\int_{\Omega}|\nabla u|^{2} d \Omega
$$

and $u \in W^{1,2}(\Omega, X)$ (or $u$ is finite energy) if $E(u)<\infty$. Let $\bar{x}(x, t)$ be the flow generated by $Z \in \Gamma(T \bar{\Omega})$. Then for a.e. $x \in \Omega$,

$$
\left|u_{*}(V)\right|(x)=\lim _{\sigma \rightarrow 0} \frac{d(u(x), u(\bar{x}(x, \epsilon)))}{\epsilon}
$$

and

$$
|\nabla u|^{2}=\int_{S^{n-1}}\left|u_{*}(\omega)\right|^{2} d \Sigma
$$

where $S^{n-1} \subset T_{x} \Omega$. For a Lipschitz Riemannian domain $\Omega$ and $u \in W^{1,2}(\Omega, X)$, there is a well-defined trace map $\operatorname{tr}(u) \in L^{2}(\partial \Omega, X)$. With this and assuming an upper curvature bound, the Dirichlet Problem can be solved:

Theorem 2.3 ([KS1], [S1], S2]). Let $(\Omega, g)$ be a Lipschitz Riemannian domain with boundary and let $(X, d)$ be a complete metric space of curvature bounded from above. Let $\phi \in W^{1,2}(\Omega, X)$. Define

$$
W_{\phi}^{1,2}=\left\{u \in W^{1,2}(\Omega, X) \mid \operatorname{tr}(u)=\operatorname{tr}(\phi)\right\} .
$$

Then there exists $u$ such that

$$
E(u)=\inf _{v \in W_{\phi}^{1,2}} E(v)
$$

Furthermore, $u$ is locally Lipschitz continuous in the interior and Hölder continuous up to the boundary. $u$ is called a harmonic map.

Furthermore, (still assuming an upper curvature bound) we can make sense of the notion of the pull back inner product

$$
\pi: \Gamma(T \bar{\Omega}) \times \Gamma(T \bar{\Omega}) \rightarrow L^{1}(\bar{\Omega})
$$

for any map $u \in W^{1,2}(\Omega, X)$ defined by

$$
\pi(V, W)=\frac{1}{4}\left|u_{*}(V+W)\right|^{2}-\frac{1}{4}\left|u_{*}(V-W)\right|^{2} \quad \text { for } V, W \in \Gamma(T \bar{\Omega}) .
$$

Hence, for $u \in W^{1,2}(D, X)$ where $D$ is the unit disk in the plane, we can define the area as

$$
A(u)=\int_{D} \sqrt{\operatorname{det}(\pi)} d z .
$$

Using the variational tools developed in [KS1], we can solve the Plateau Problem in this setting (a complete proof which is a straightforward extension of an argument due to Morrey $\mathrm{Mo}$ is contained in $[\mathrm{Me} 2$ ) and we have: 
The Plateau Problem. Let $\Gamma$ be a closed Jordan curve in $X$ where $X$ is a complete locally compact metric space of curvature bounded from above by $\kappa$ (with length of $\Gamma$ less than $\frac{\pi}{\sqrt{\kappa}}$ if $\kappa>0$ ) and let

$$
C_{\Gamma}=\left\{u \in W^{1,2}(D, X):\left.u\right|_{\partial D} \text { parametrizes } \Gamma \text { monotonically }\right\} .
$$

There exists $u \in C_{\Gamma}$ so that $A(u)=\inf \left\{A(v) \mid v \in C_{\Gamma}\right\}$. Moreover, $u$ is weakly conformal, i.e. $\pi_{11}=\pi_{22}$ and $\pi_{12}=0=\pi_{21}$ and Lipschitz continuous in the interior of $D$ and continuous up to the boundary.

We call $\lambda=\pi_{11}$ the conformal factor of the pull back inner product. Recall in the case when $X$ is a smooth Riemannian manifold, the Gauss curvature of the minimal surface is expressed by

$$
K=\frac{1}{2 \lambda} \triangle \log \lambda .
$$

Hence the following theorem in [Me1] says that the curvature of the minimal surface is bounded from above by $\kappa$ when $X$ is a metric space of curvature bounded from above by $\kappa$.

Theorem 2.4. Let $u: D \rightarrow X$ be a conformal harmonic map with conformal factor $\lambda$. The function $\log \lambda$ is in $W_{\text {loc }}^{1,1}(D)$ and satisfies

$$
\int_{\Omega} \log \lambda \triangle \phi \geq-2 \kappa \int_{\Omega} \lambda \phi
$$

for any $\phi \in C_{c}^{\infty}(\Omega)$.

Using the properties of subharmonic functions discussed below, the above theorem also implies that $\lambda(z)>0$ for a.e $z \in \Omega$. In fact, in [Me1] we show:

Theorem 2.5. Let $u \in W^{1,1}(D, X)$ be a conformal harmonic map with conformal factor $\tilde{\lambda}$. Then $\tilde{\lambda}>0$ for a.e. $z \in D$. Furthermore, there is a representative $\lambda$ in the $L^{1}$-equivalence class of the function $\tilde{\lambda}$ defined by

$$
\lambda(z)=\lim _{r \rightarrow 0} \frac{1}{\pi r^{2}} \int_{D_{r}(z)} \tilde{\lambda}(x) d x
$$

where $D_{r}(z)$ is a disk of radius $r$ around $z$ so that the perimeter of the set $\{z$ : $\lambda(z)<t\}$ goes to zero as $t \rightarrow 0$.

For the remainder of the paper, when we say " $\lambda$ is the conformal factor of a map $u$ ", we mean the function defined by (3).

2.3. Subharmonic Functions. The theory of subharmonic functions is important in the study of minimal surfaces in NPC spaces so we review the definitions.

Definition 2.6. Let $\Omega$ be a domain in $\mathbf{R}^{n}$. A function $f \in W_{l o c}^{1,1}(\Omega)$ is said to be weakly subharmonic if for every non-negative $\varphi \in C_{c}^{\infty}(\Omega)$,

$$
0 \leq \int_{\Omega}-\nabla \varphi \cdot \nabla f d x
$$

The following definition is due to Riesz (see $[\mathrm{HK}]$ ). 
Definition 2.7. A function $f$ defined in a domain $\Omega \subset \mathbf{R}^{n}$ is said to be subharmonic in $\Omega$ if

(i) $-\infty \leq f(x)<\infty$ in $\Omega$.

(ii) $f(x)$ is upper semicontinuous in $\Omega$.

(iii) If $x_{0}$ is any point in $\Omega$, then there exists arbitrarily small positive values of $r$ such that

$$
f\left(x_{0}\right) \leq \frac{1}{c_{n} r^{m-1}} \int_{\partial B_{r}\left(x_{0}\right)} f(x) d \sigma(x)
$$

where $d \sigma(x)$ denotes surface area on $\partial B_{r}\left(x_{0}\right)$ and $c_{n}$ is a dimensional constant.

It is known that if $f$ is a weakly subharmonic map, there is an $L^{1}$-representative of $f$ which is subharmonic and if $f$ is upper semicontinuous and weakly subharmonic, then $f$ is subharmonic. The following is due Cartan (see [HK], Theorem 5.32):

Theorem 2.8. Let $f(x)$ be a subharmonic function not identically $-\infty$ in a domain $\Omega \subset \mathbf{R}^{n}$. Then the subset $E$ of $D$ where $f(x)=-\infty$ is a $G_{\delta}$ set of capacity zero.

\section{Monotonicity of the Boundary Map}

For any set $S \subset X$ and any $P \in X$, the distance from $P$ to $S$ is defined $d(P, S)=$ $\inf _{Q \in S} d(P, Q)$. Let $\mathcal{C}$ be a closed convex set. Then for each point $P \in X$ there exists a unique point $Q \in \mathcal{C}$ such that $d_{P Q}=d(P, \mathcal{C})$ as can be seen from the following: the existence of $Q$ is guaranteed by the fact that $\mathcal{C}$ is closed. Suppose $Q^{\prime} \in \mathcal{C}$ is another point so that $d_{P Q^{\prime}}=d(P, \mathcal{C})$. By convexity, $\overline{Q Q^{\prime}} \subset \mathcal{C}$. Denoting by $Q_{t}$ the point on $\overline{Q Q^{\prime}}$ with $d_{Q Q_{t}}=t d_{Q Q^{\prime}}$, inequality (1) gives

$$
d_{P Q_{t}}^{2} \leq(1-t) d_{P Q}+t d_{P Q^{\prime}}-t(1-t) d_{Q Q^{\prime}},
$$

and hence $d_{P Q_{t}}<d_{P Q}=d_{P Q^{\prime}}$ for $0<t<1$, and this is a contradiction. Therefore, we can define a projection map $\pi: X \rightarrow \mathcal{C}$ by setting $\pi(P)=Q$. We claim that for each $P, Q \in X$,

$$
d(\pi(P), \pi(Q)) \leq d(P, Q) .
$$

To see this, we set $R=\pi(P)$ and $S=\pi(Q)$ and let $\{\bar{P}, \bar{Q}, \bar{R}, \bar{S}\}$ be a subembedding of $\{P, Q, R, S\}$. By convexity, $\overline{R S} \subset \mathcal{C}$ and hence $\angle(P S R), \angle(Q R S) \geq \frac{\pi}{2}$; otherwise, we contradict the fact that $d_{P S}=d(P, \mathcal{C})$ and $d_{Q R}=d(P, \mathcal{C})$. Therefore, $\angle(\bar{P} \bar{S} \bar{R}), \angle(\bar{Q} \bar{R} \bar{S}) \geq \frac{\pi}{2}$ which implies $d_{P Q}=|\bar{P}-\bar{Q}| \geq|\bar{R}-\bar{S}|=d_{R S}$.

Lemma 3.1. Let $u: M \rightarrow X$ be a harmonic map. Then $u(M)$ is contained in the convex hull of $u(\partial M)$.

Proof. Let $\mathcal{C}$ be the convex hull of $u(\partial M)$ and $\pi: X \rightarrow \mathcal{C}$ be a projection map. For every $x, y \in M$, we have $d(\pi \circ u(x), \pi \circ u(y)) \leq d(u(x), u(y))$ by equation (4). Hence $E(\pi \circ u) \leq E(u)$. Harmonic maps for a given boundary data are unique in an NPC space (see [KS1]), so this implies $\pi \circ u=u$.

Lemma 3.2. Let $u: M \rightarrow X$ be a weakly conformal harmonic map and $r: M \rightarrow \mathbf{R}$ be defined by $r(x)=d(u(x), Q)$ for some point $Q \in X$. The function $\log r$ is in $W_{\text {loc }}^{1,1}(M)$ and is weakly subharmonic, i.e. for every non-negative $\varphi \in C_{c}^{\infty}(M)$,

$$
-\int_{M} \nabla \varphi \cdot \nabla \log r^{2} \geq 0
$$


Proof. Using a partition of unity argument, it suffices to prove this inequality locally. Furthermore, for the invariance of harmonicity with respect to a conformal change of coordinates, it is sufficient to prove the inequality where $u: D \rightarrow X$ and $D$ is a unit disk in the complex plane.

Let $r: D \rightarrow X$ be defined by $r(x)=d(u(x), Q)$. If $u$ is harmonic, then $u$ is Lipschitz in the interior of $D$, thus so is $r . r$ satisfies the following weak inequality:

$$
-\int_{D} \nabla \varphi \cdot \nabla r^{2} \geq 2 \int_{D} \varphi|\nabla u|^{2}
$$

for every non-negative $\varphi \in C_{c}^{\infty}(M)$. The above inequality is due to Gromov and Schoen [GS] in the case when $X$ is a Riemannian simplicial complex of non-negative curvature. In the general case where $X$ is an NPC space, the proof of this inequality is contained in [S1] where it is credited to Korevaar and Schoen.

Since $r$ is Lipschitz, $\nabla r$ exists a.e. Let $A=\{x \in D: \nabla r$ exists and $|\nabla r| \neq 0\}$ and let $W=\frac{\nabla r(x)}{|\nabla r(x)|}$ for $x \in A$. By the triangle inequality and the conformality of $u$,

$$
\begin{aligned}
|\nabla r|^{2} & =\lim _{\epsilon \rightarrow 0} \frac{|r(x+\epsilon W)-r(x)|^{2}}{\epsilon^{2}} \\
& \leq \lim _{\epsilon \rightarrow 0} \frac{d^{2}(u(x+\epsilon W), u(x))}{\epsilon^{2}} \\
& =\frac{1}{2}|\nabla u|^{2}
\end{aligned}
$$

for a.e. $x \in D$. Thus,

$$
-\int_{D} \nabla \varphi \cdot \nabla r^{2} \geq 4 \int_{D} \varphi|\nabla r|^{2} .
$$

Let $r_{\epsilon}=r+\epsilon$. Since $r \in H_{l o c}^{1}(D)$, we have that $\log r_{\epsilon} \in H_{l o c}^{1}(D)$. Furthermore, since $\nabla r=\nabla r_{\epsilon}$ whenever $\nabla r$ exists,

$$
-\int_{D} \nabla \varphi \cdot \nabla r_{\epsilon}^{2} \geq 4 \int_{D} \varphi\left|\nabla r_{\epsilon}\right|^{2}
$$

Noting that $\frac{\varphi}{r_{\epsilon}^{2}} \in H_{l o c}^{1}(D)$ and hence is an admissible function for $\varphi$ in inequality (17), we have

$$
\begin{aligned}
-\int_{D} \nabla \varphi \cdot \nabla \log r_{\epsilon}^{2} & =-\int_{D} \nabla \varphi \cdot \frac{\nabla r_{\epsilon}^{2}}{r_{\epsilon}^{2}} \\
& \geq-\int_{D} \nabla\left(\frac{\varphi}{r_{\epsilon}^{2}}\right) \cdot \nabla r_{\epsilon}^{2}-2 \int_{D} \frac{\varphi}{r_{\epsilon}^{3}} \nabla r_{\epsilon} \cdot \nabla r_{\epsilon}^{2} \\
& \geq 4 \int_{D} \frac{\varphi}{r_{\epsilon}^{2}}\left|\nabla r_{\epsilon}\right|^{2}-4 \int_{D} \frac{\varphi}{r_{\epsilon}^{2}}\left|\nabla r_{\epsilon}\right|^{2} \\
& =0 .
\end{aligned}
$$

Since $r$ is a continuous weakly subharmonic function, $r(x) \neq 0$ for a.e. $x$. Furthermore, $r(x) \leq L$ for some $L$ by the convex hull property (Lemma 3.1). Hence Theorem 3.2 follows from the following claim:

Claim. Let $f_{n}: D \rightarrow \mathbf{R}$ be a decreasing sequence of functions converging to a non-negative $f$ not identically equal to 0 in any open set. Assume $f_{n} \leq M$ for 
$n=1,2, \ldots$. If

$$
\int_{D} \log f_{n} \triangle \varphi \geq 0
$$

for every non-negative $\varphi \in C_{c}^{\infty}(D)$, then

$$
\int_{D} \log f \triangle \varphi \geq 0 .
$$

Proof. W.l.o.g. assume $f(0) \neq 0$. Then

$$
\begin{aligned}
-\infty & <\log f(0) \\
& \leq \log f_{n}(0) \\
& \leq \frac{1}{\pi} \int_{D} \log f_{n} \\
& \leq \log M .
\end{aligned}
$$

Let $F_{n}=\log M-\log f_{n}$ and $F=\log M-\log f . F_{n}$ is an increasing sequence of non-negative functions. By the Monotone Convergence Theorem,

$$
\lim _{n \rightarrow \infty} \int_{D} F_{n}=\int_{D} F
$$

in other words,

$$
\lim _{n \rightarrow \infty} \int_{D} \log f_{n}=\int_{D} \log f .
$$

In particular, $\log f \in L^{1}(D)$. Thus for a non-negative $\varphi \in C_{c}^{\infty}(D)$, we have that $\log f_{n} \triangle \varphi \rightarrow \log f \triangle \varphi$ a.e. Since $\left|\log f_{n} \triangle \varphi\right| \leq|\triangle \varphi|_{\infty}\left|\log f_{n}\right|$, by the the Dominated Convergence Theorem, we have the desired result.

This allows us to prove:

Theorem 3.3. Let $M$ be a smooth compact Riemann surface with boundary and $u: M \rightarrow X$ be a weakly conformal harmonic map. Then $u$ does not map any boundary arc of $M$ to a single point.

Proof. Suppose there is an open set $I \subset \partial M$ so that $u(I)=Q$. By the invariance of harmonicity by conformal change of coordinates, we can construct a weakly conformal harmonic map $u: D^{+} \rightarrow X$ where $D^{+}$is the upper half of the unit disk and $u(x, 0)=Q$. Thus if $r(x, y)=d(u(x, y), Q)$, then $r(x, 0)=0$ for $-1<x<1$. By Lemma 3.2, $\log r$ is weakly subharmonic in $D^{+}$. We extend $r$ to all of $D=$ $\left\{(x, y) \in \mathbf{R}^{2}: x^{2}+y^{2}<1\right\}$ by reflection, i.e., $r(-x, y)=r(x, y)$. We check that $\log r: D \rightarrow \mathbf{R}$ is subharmonic (in the sense of Riesz): clearly $-\infty \leq \log r<\infty$ and, additionally, $\log r$ is upper semicontinous since it is a decreasing limit of continuous functions $\log \left(r+\epsilon_{i}\right), \epsilon_{i} \rightarrow 0$. At a point $(x, y)$ with $y \neq 0$, (iii) of Definition 2.7 follows immediately from equation (5). If $y=0$, this is also trivially true since $\log r(x, 0)=-\infty$. But this contradicts Theorem 2.8 since we show that $\log r$ is a subharmonic function with $\log r(x, 0)=-\infty$ for $-1<x<1$.

\section{ISOPERIMETRIC INEQUALITY}

Let $(M, g)$ be a compact Riemann surface with boundary and $u: M \rightarrow X$ be a weakly conformal harmonic map with conformal factor $\lambda . \lambda$ is a locally defined function and if $x, y$ are local conformal coordinates, $\lambda d x d y$ is the volume form pulled 
back by the map $u$ (well-defined because of the conformal invariance of the energy). The area of the minimal surface $u(M)$ is defined as

$$
A=\int_{M} \lambda d x d y
$$

It is shown in [Me1] that the distance function $d_{\lambda}$ on $M$ induced by the conformal factor $\lambda$ makes $\left(M, d_{\lambda}\right)$ into an NPC space. The following proof of the isoperimetric inequality is an adaptation of an argument due to Michael and Simon [MS]. In [MS], the ambient space is $\mathbf{R}^{n}$ and the submanifold has arbitrary dimension $m, m<n$. Here we consider $m=2$ and the ambience space $X$ is an NPC space. The technical modification we must make to carry through the proof of [MS] is that (instead of working directly on the submanifold) we must work in the domain space $M$ with the induced volume form $\lambda d x d y$.

Lemma 4.1. Let

$$
S_{\rho}\left(x_{0}\right)=\left\{x \in M \mid d\left(u(x), u\left(x_{0}\right)\right)<\rho\right\}
$$

and

$$
A(\rho)=\int_{S_{\rho}\left(x_{0}\right)} \lambda d x d y
$$

Then

$$
\frac{A(\rho)}{\rho^{2}} \geq \pi
$$

for sufficiently small $\rho$.

Proof. Let $B_{\rho}\left(x_{0}\right)$ be a geodesic ball of radius $\rho$ around $x_{0}$ in distance function $d_{\lambda}$. We let

$$
A_{\lambda}(\rho)=\int_{B_{\rho}\left(x_{0}\right)} \lambda d x d y
$$

By choosing $\rho$ sufficiently small, we can work in a conformal coordinate patch $U$. For any piecewise $C^{1}$ curve $\sigma:[0,1] \rightarrow M$ from $x$ to $x_{0}$, equation (1.9xvi) of KS1] gives

$$
\int_{0}^{1} \sqrt{\lambda(\sigma(t))}\left|\sigma^{\prime}(t)\right| d t=\lim _{|\triangle| \rightarrow 0} \Sigma d\left(u\left(t_{i}\right), u\left(t_{i+1}\right)\right) .
$$

By the triangle inequality, we see that $d\left(u\left(x_{0}\right), u(x)\right) \leq d_{\lambda}\left(x_{0}, x\right)$. Hence, $B_{\rho}\left(x_{0}\right) \subset$ $S_{\rho}\left(x_{0}\right)$ and $A_{\lambda}(\rho) \leq A(\rho)$. Thus, it suffices to prove $\frac{A_{\lambda}(\rho)}{\rho^{2}} \geq \pi$.

Let $\epsilon>0$ be given. There exists smooth functions $\lambda^{\sigma}$ so that $\lambda^{\sigma} \geq \lambda, \sqrt{\lambda^{\sigma}} \rightarrow \sqrt{\lambda}$ in $H_{l o c}^{1}(M)$ and $\lambda^{\sigma}\left(d x^{2}+d y^{2}\right)$ defines a non-positively curved metric away from the boundary of $M$. (The functions $\lambda^{\sigma}$ is obtained by mollifying $\log \lambda$ by a symmetric mollifying function to obtain $(\log \lambda)_{\sigma}$ and setting $\lambda^{\sigma}=e^{(\log \lambda)_{\sigma}}$. See [Me1].) Thus, there exists $\sigma_{1}>0$ such that for all $\sigma<\sigma_{1}$ and any $K \subset U$,

$$
0 \leq \int_{K} \lambda^{\sigma}-\int_{K} \lambda<\epsilon
$$

Furthermore, since $\lambda \in L^{1}(M)$, there exists $\delta>0$ such that for any set $A \subset M$ with measure less than $\delta$,

$$
\int_{A} \lambda \leq \epsilon
$$


Let $B_{\rho}^{\sigma}\left(x_{0}\right)$ be the geodesic ball of radius $\rho$ with respect to metric $\lambda^{\sigma}\left(d x^{2}+d y^{2}\right)$. Noting that $B_{\rho}^{\sigma}\left(x_{0}\right) \subset B_{\rho}\left(x_{0}\right)$, there exists $\sigma_{2}>0$ such that for all $\sigma<\sigma_{2}$,

$$
0 \leq \int_{B_{\rho}\left(x_{0}\right)-B_{\rho}^{\sigma}\left(x_{0}\right)} \lambda<\epsilon .
$$

Therefore, for $\sigma<\min \left\{\sigma_{1}, \sigma_{2}\right\}$

$$
\begin{aligned}
\left|\int_{B_{\rho}^{\sigma}\left(x_{0}\right)} \lambda^{\sigma}-\int_{B_{\rho}\left(x_{0}\right)} \lambda\right| & \leq\left|\int_{B_{\rho}^{\sigma}\left(x_{0}\right)} \lambda^{\sigma}-\int_{B_{\rho}^{\sigma}\left(x_{0}\right)} \lambda\right|+\left|\int_{B_{\rho}^{\sigma}\left(x_{0}\right)} \lambda-\int_{B_{\rho}\left(x_{0}\right)} \lambda\right| \\
& <2 \epsilon .
\end{aligned}
$$

Since $\lambda^{\sigma}\left(d x^{2}+d y^{2}\right)$ defines a smooth surface of non-positive curvature,

$$
\int_{B_{\rho}^{\sigma}\left(x_{0}\right)} \lambda^{\sigma} \leq \pi \rho^{2}
$$

and hence

$$
\int_{B_{\rho}\left(x_{0}\right)} \lambda \leq \pi \rho^{2}+2 \epsilon
$$

Since $\epsilon$ is arbitrary,

$$
\int_{B_{\rho}\left(x_{0}\right)} \lambda \leq \pi \rho^{2}
$$

Since $A(\rho) \geq A_{\lambda}(\rho)$, we have proved the lemma.

Lemma 4.2. Suppose $\xi \in C^{1}(-\infty, \infty)$ is a non-decreasing function such that $\xi(t)=0$ for $t \leq 0$ and $h \in C_{0}^{1}(M)$ is a non-negative function. For $x_{0} \in M$, define

$$
\varphi_{x_{0}}(\rho)=\int_{M} h(x) \xi(\rho-r(x)) \lambda(x) d x d y
$$

and

$$
\phi_{x_{0}}(\rho)=\int_{M}|\nabla h|(x) \xi(\rho-r(x)) \lambda^{1 / 2}(x) d x d y
$$

where $r(x)=d\left(u(x), u\left(x_{0}\right)\right)$. Then

$$
-\frac{d}{d \rho}\left(\frac{\varphi_{x_{0}}(\rho)}{\rho^{2}}\right) \leq \frac{\phi_{x_{0}}(\rho)}{\rho^{2}} .
$$

Proof. We can follow the proof of Lemma 2.2 of [MS] with some technical modification in the beginning. In our setting we use equation (6) to see that for any $\Psi \in C^{1}(M)$,

$$
\begin{aligned}
4 \int \Psi(x) \lambda(x) d x d y & \leq-\int \nabla \Psi(x) \cdot \nabla\left(r^{2}(x)\right) d x d u \\
& =-2 \int r(x) \nabla \Psi(x) \cdot \nabla r(x) d x d y
\end{aligned}
$$

Letting $\Psi(x)=h(x) \xi(\rho-r(x))$, we have

$$
\nabla \Psi(x)=-h(x) \xi^{\prime}(\rho-r(x)) \nabla r(x)+\xi(\rho-r(x)) \nabla h(x)
$$


whenever $\nabla r$ exists (recall that it exists a.e. since $r$ is a Lipschitz map). Consequently,

$$
\begin{aligned}
2 \int h(x) \xi(\rho-r(x)) \lambda(x) d x d y \leq & \int r(x) h(x) \xi^{\prime}(\rho-r(x))|\nabla r(x)|^{2} d x d y \\
& -\int r(x) \xi(\rho-r(x)) \nabla h(x) \cdot \nabla r(x) d x d y \\
\leq & \int r(x) h(x) \xi^{\prime}(\rho-r(x)) \lambda d x d y \\
& +\int r(x) \xi(\rho-r(x))|\nabla h| \lambda^{\frac{1}{2}}(x) d x d y
\end{aligned}
$$

We are done since we have shown $2 \varphi_{x_{0}}(\rho)-\rho \varphi_{x_{0}}^{\prime}(\rho) \leq \rho \phi_{x_{0}}(\rho)$.

Lemma 4.3. Let $h \in C_{0}^{1}(M)$ be such that $h\left(x_{0}\right) \geq 1$. Define

$$
\bar{\varphi}_{x_{0}}(\rho)=\int_{S_{\rho}\left(x_{0}\right)} h(x) \lambda(x) d x d y
$$

and

$$
\bar{\phi}_{x_{0}}(\rho)=\int_{S_{\rho}\left(x_{0}\right)}|\nabla h|(x) \lambda^{1 / 2}(x) d .
$$

Then there exists $\rho>0$ such that

$$
\rho<2\left(\frac{1}{\pi} \int_{M} h(x) \lambda(x) d x d y\right)^{1 / 2}
$$

and

$$
\bar{\varphi}_{x_{0}}(4 \rho) \leq 16\left(\frac{1}{\pi} \int_{M} h(x) \lambda(x) d x d y\right)^{1 / 2} \bar{\psi}_{x_{0}}(\rho) .
$$

Proof. We can follow the proof of [MS], Lemma 2.3 replacing $d \mu$ by $\lambda d x d y$.

We are now ready to prove

Theorem 4.4. Let $X$ be a NPC space. There exists an universal constant $C>0$ so that $L^{2} \geq C A$ for any weakly conformal harmonic map $u: M \rightarrow X$ from a compact Riemann surface with boundary. Here, $L$ is the length of $u(\partial M)$ and $A$ is the area of the minimal surface, i.e. $A=\int_{M} \lambda d x d y$ where $\lambda$ is the conformal factor of $u$.

Proof. We can follow the covering argument of [MS], Theorem 2.1 to show

$$
\left(\int_{M} h^{2} \lambda d x d y\right)^{\frac{1}{2}} \leq \frac{32}{\sqrt{\pi}} \int_{M}|\nabla h| \lambda^{\frac{1}{2}} d x d y
$$

for any $h \in C^{1}(M)$.

Let $\Gamma$ be a set of curves close to $\partial M$ so that $M-\Gamma$ consists of a connected component $M^{\prime}$ disjoint from $\partial M$ and a set of annuli, each annulus forming a neighborhood around a component of $\partial M$. Fix $\epsilon>0$. Since $u$ is uniformally continuous and $\lambda$ is integrable, we can choose $\Gamma$ so that

$$
|\operatorname{length}(u(\Gamma))-L|<\frac{\sqrt{\pi}}{32} \epsilon
$$


and

$$
\left(\int_{M} \lambda d x d y\right)^{1 / 2}-\left(\int_{M^{\prime}} \lambda d x d y\right)^{1 / 2}<\epsilon
$$

By equation (1.9xvi) of [KS1], we can assume that

$$
\operatorname{length}(u(\Gamma))=\int_{\Gamma} \lambda^{1 / 2}
$$

Let $\lambda^{\sigma}$ be as in the proof of Lemma 4.1 and recall that $\lambda^{\sigma} \geq \lambda$. Thus, for any $h \in C_{c}^{\infty}(M)$ and sufficiently small $\sigma$,

$$
\int_{M}|\nabla h| \lambda^{1 / 2} d x d y \leq \int_{M}|\nabla h|\left(\lambda^{\sigma}\right)^{1 / 2} d x d y
$$

and

$$
\int_{M} h^{2} \lambda^{\sigma} d x d y \leq \int_{M} h^{2} \lambda d x d y+|h|_{\infty}^{2} \int_{M}\left(\lambda^{\sigma}-\lambda\right) d x d y .
$$

Consequently, for any $h \in C_{c}^{\infty}(M)$ with $|h|_{\infty} \leq 1$, we have

$$
\left(\int_{M} h^{2} \lambda^{\sigma} d x d y\right)^{1 / 2} \leq \frac{32}{\sqrt{\pi}} \int_{M}|\nabla h|\left(\lambda^{\sigma}\right)^{1 / 2} d x d y+O(\sigma)
$$

where $O(\sigma) \rightarrow 0$ as $\sigma \rightarrow 0$. For a fixed $\sigma>0$ sufficiently small and $\delta$ sufficiently small, let $A_{\delta} \subset M$ be a $\delta$-neighborhood of $\Gamma$ where the distance is measured in the (smooth) metric $\lambda^{\sigma}\left(d x^{2}+d y^{2}\right)$. Letting $h$ approximate a function which is identically 1 in $M^{\prime}-A_{\delta}$ and which slopes down to zero in $A_{\delta}$ and letting $\delta \rightarrow 0$, we have

$$
\left(\int_{M^{\prime}} \lambda^{\sigma} d x d y\right)^{1 / 2} \leq \frac{32}{\sqrt{\pi}} \int_{\Gamma}\left(\lambda^{\sigma}\right)^{1 / 2}+O(\sigma) .
$$

Now letting $\sigma \rightarrow 0$ and using the fact that $\sqrt{\lambda^{\sigma}} \rightarrow \sqrt{\lambda}$ in $H_{l o c}^{1}$, we have

$$
\left(\int_{M^{\prime}} \lambda d x d y\right)^{1 / 2} \leq \frac{32}{\sqrt{\pi}} \int_{\Gamma} \lambda^{1 / 2}
$$

Hence,

$$
A^{1 / 2} \leq \frac{32}{\sqrt{\pi}} L+2 \epsilon
$$

Since $\epsilon$ is arbitrary, we are done.

\section{REFERENCES}

[ABN] A.D. Alexandrov, V.N. Berestoskii and I.G.Nikolaev. Generalized Riemann Spaces. Uspekhi Mat. Nauk, 41 (1986), 3-44. MR 88e:53103

[C] K. Corlette. Archimedian Superrigidity and Hyperbolic Geometry. Ann. of Math., 135 (1992) 165-182. MR 92m:57048

[GS] M. Gromov and R. Schoen. Harmonic Maps into Singular Spaces and p-adic Supperrigidity for Lattices in Groups of Rank One. Inst. Hautes Études Sci. Publ. Math., 76 (1992), 165246. MR 94e:58032

[HL] R. Hardt and F-H Lin. Harmonic Maps into Round Cones and Singularities of Nematic Liquid Crystals. Math Z., 213 (1993) no. 4, 575-593. MR 94h:58062

[HK] W.K. Hayman and P.B. Kennedy. Subharmonic Functions, vol. 1. Academic Press, London, 1976. MR 57:665

[J1] J. Jost. Equilibrium Maps Between Metric Spaces. Cal. Var. Partial Differential Equations 2, (1994) 173-204. MR 98a:58049

[J2] J. Jost. Convex Functionals and Generalized Harmonic Maps into Spaces of Non-positive Curvature. Comment. Math. Helv., 70 (1995) no. 4, 659-673. MR 96j:58043 
[KS1] N. Korevaar and R. Schoen. Sobolev Spaces and Harmonic Maps for Metric Space Targets. Communications in Analysis and Geometry, 1 (1993), 561-659. MR 95b:58043

[KS2] N. Korevaar and R. Schoen. Global Existence Theorems for Harmonic Maps to Non-locally Compact Spaces. Comm. Anal. Geom., 5 (1997), 333-387. MR 99b:58061

[Me1] C. Mese. The Curvature of Minimal Surfaces in Singular Spaces. to appear in Communications of Analysis and Geometry.

[Me2] C. Mese. Minimal Surfaces and Conformal Mappings into Singular Spaces. Ph.D. thesis. Stanford University, 1996.

[MS] J.H. Michael and L.M. Simon. Sobolev and Mean-Value Inequalities on Generalized Submanifolds of $\mathbf{R}^{n}$. Comm. Pure Appl. Math. 26 (1973), 361-379. MR 49:9717

[Mo] C.B. Morrey. The Plateau Problem on a Riemannian Manifold. Annals of Mathematics, 49 (1948), 807-851. MR 10:259f

[N] I.G. Nikolaev. Solution of the Plateau Problem in Spaces of Curvature at most K. Sibirsk. Mat. Zh., 20 (1979), 345-353. MR 80k:58041

[R1] Y.G. Reshetnyak. Geometry IV Non-regular Riemann Geometry. Translated by E. Primrose. Encyclopedia of Mathematical Science, 70. Springer-Berlag, Berlin, 1993.

[R2] Y.G. Reshetnyak. Nonexpanding Maps in a Space of Curvature No Greater than K. Sibirsk. Mat. Ž. 9 (1968), 918-927. MR 39:6235

[S1] T. Serbinowski. Boundary Regularity of Harmonic Maps to Non-positively Curved Metric Spaces. Comm. Anal. Geom., 2 (1994), 139-153. MR 95k:58050

[S2] T. Serbinowski. Harmonic Maps into Metric Spaces with Curvature Bounded Above. Ph.D. thesis. University of Utah, 1995.

[W1] M. Wolf. Harmonic Maps from surfaces to R-trees. Math. Z., 218 no. 4 (1995), 577-593. MR 97b:58042

[W2] M. Wolf. On Realizing Measured Foliations Via Quadratic Differentials of Harmonic Maps to R-trees. J. Anal. Math., 68 (1996), 107-120. MR 97k:32032

Department of Mathematics, University of Southern California, Los Angeles, CalIFORNIA 90089

Current address: Department of Mathematics, Connecticut College, New London, Connecticut 06320

E-mail address: cmes@conncoll.edu 